\title{
Adhesion and Co-stimulatory Molecules in the Pathogenesis of Hepatic and Intestinal Schistosomiasis Mansoni
}

\author{
Werner Jacobs* , Eric Van Marck ${ }^{+}$
}

Department of Pathology, University of Antwerp, Universiteitsplein 1, B-2610 Wilrijk, Belgium

\begin{abstract}
Infection of a susceptible host with the blood fluke Schistosoma mansoni results in the formation of periovular granulomas and subsequent fibrosis in the target organs. Granulomogenesis and fibrogenesis are mediated by immunological events which require cell-cell and cell-matrix interactions. In this review, the role of adhesion and co-stimulatory molecules in the genesis of the schistosomal pathology (granulomogenesis and fibrogenesis) is outlined. These molecules provide essential immunological interactions not only for the initiation of granuloma formation but also for the maintenance and modulation of the schistosomal granuloma during chronic infection. Furthermore, the role of secreted soluble adhesion molecules in the different clinical forms and in the modulation of the schistosomal granuloma is discussed. Recent new insights into the role of adhesion molecules for the induction of pathology by other developmental stages of the parasite (other than eggs) will be presented.
\end{abstract}

Key words: adhesion molecules - co-stimulatory molecules - Schistosoma - pathogenesis - granuloma formation

\section{SCHISTOSOMIASIS}

Schistosomiasis is a helminthic disease, which causes considerable morbidity and mortality worldwide (WHO 1993). Infection of a susceptible host with the blood fluke Schistosoma mansoni results in the release of parasite eggs in the mesenteric and portal venous system, leading to entrapment of these eggs in the perisinusoidal spaces of the liver and in the intestinal wall. Tissue localization of antigen-secreting eggs initiates a persistent immunological insult to which the host responds by marshalling leukocyte recruitment and activation of inflammatory and immune-mediated responses resulting in granuloma formation and subsequent fibrosis. In a subpopulation of infected individuals, chronic granulomatous inflammation and fibrosis may cause hepato-splenomegaly, portal hypertension, and the development of oesophageal varices that may rupture and cause fatal bleeding, this being the main cause of death in this disease. During chronic infection, schistosome granulomas become "modulated" resulting in smaller hepatic and colonic granulomas (Colley 1975). Clinically, chronic $S$. mansoni infection in humans presents as a spec-

This work was supported by grant 1.5.043.96 $\mathrm{N}$ of the Fonds voor Wetenschappelijk Onderzoek (F.W.O.), Vlaanderen.

*Research Assistant of the F.W.O., Vlaanderen

${ }^{+}$Corresponding author. Fax: +32-3-821.47.53. E-mail: eric.van.marck@uza.uia.ac.be

Received 17 March 1998

Accepted 7 May 1998 trum of disease forms, ranging from the relatively mild intestinal through the hepato-intestinal form and at the other end of the spectrum the severe hepatosplenic disease. The murine model of experimental schistosomiasis has been widely studied because it provides a unique model for the study towards the pathogenetic mechanisms underlying both granulomogenesis and fibrogenesis. The granulomogenic process in schistosomiasis is dependent on CD4+ T helper (Th) lymphocytes (Wynn \& Cheever 1995) and results in a shift of the immunologic balance from a Th1 to a Th2 cell type response (Pearce et al. 1991, Grzych et al. 1991).

\section{ADHESION AND CO-STIMULATORY MOLECULES}

The emigration of circulating leukocytes into tissues and migration towards an antigenic nidus is a characteristic of the inflammatory response. Extravasation of leukocytes from the blood stream is controlled by the expression of cell surface adhesion molecules on both the circulating cells and on the vascular endothelium. Further immune responsiveness of T-cells to antigens and the effector activities of leukocytes requires cell surface molecules mediating cell-cell and cell-matrix interactions and providing additional co-stimulatory activity.

Cellular adhesion molecules are currently classified into three families. In the immunoglobulin superfamily, the cell adhesion molecules are built up of "immunoglobulin-like" domains. Attention has been drawn to a very important feature of the immunoglobulin domain structure, namely mutual complementarity which allows strong interdomain 
noncovalent interactions. Major histocompatibility complex (MHC) molecules, the adhesion molecules ICAM-1, VCAM-1, and the co-stimulatory molecules B7/1 and B7/2 are some of the important members of this superfamily (Springer 1990, Freeman et al. 1993). The integrin family of adhesion molecules represents a group of proteins composed of noncovalently associated $a$ and $b$ subunits. Integrins have been organized into three subfamilies based on the $b$ subunit utilization. The VLA (very late antigen) molecules (1 to 6 ) share a common b1 subunit, but different a (1 to 6) subunits. VLA molecules act as receptors that bind to extracellular matrix components (Springer 1990). VLA-4 functions both as a matrix (fibronectin) and cell (VCAM-1) receptor (Osborn et al. 1989). The LFA-1 molecule, ligand of ICAM-1, belongs to the b2 subfamily of integrins and is expressed on leukocytes. Integrins regulate leukocyte adhesion to endothelium, but where selectins, a family of $\mathrm{Ca}^{2+}$ dependent carbohydrate-binding proteins which exert functions in fast inducible intravascular adhesion and homing of lymphocytes, only mediate adhesion, integrins are still required for subsequent transendothelial migration (Johnston et al. 1989). Selectin adhesion receptors are molecules which have N-terminal lectin-like domains, an epidermalgrowth factor-like domain and varying numbers of short consensus repeats in the extracellular part of the molecule (Springer 1990, Springer \& Lasky 1991). Three selectins playing important roles in inflammatory events have so far been identified; E-, P-, and L-selectin. Selectins mediate adhesion of platelets to neutrophils and monocytes (Pselectin) (Larsen et al. 1990) or function in an early step of leukocyte binding to the endothelium, before transendothelial migration (L- and E-selectin) (Bührer et al. 1990, Springer 1990), or mediate adhesion of lymphocytes to the specialized postcapillary endothelium of lymph nodes (L-selectin) (Camerini et al. 1989). LewisX (CD15) was identified as the selectin ligand (Larsen et al. 1990, Springer \& Lasky 1991).

\section{SOLUBLE ADHESION MOLECULES IN SCHISTOSO- MIASIS PATIENTS}

Recently, soluble forms of adhesion molecules were described in human serum. One of these soluble adhesion molecules, soluble ICAM-1, has a structure consistent with a membrane-cleaved variant of cellular ICAM-1 and maintains it ligandbinding capacity (Rothlein et al. 1991, Pigott et al. 1992). Soluble forms of ICAM-1 and E-selectin were found in the serum of patients with schistosomiasis mansoni (Secor et al. 1994, LejolyBoisseau et al. 1996). Adhesion molecules can be shed by activated endothelial cells (Pigott et al.
1992, Leeuwenberg et al. 1992). Serum levels of soluble adhesion molecules in schistosomiasis patients were significantly higher when compared to control patients and differences in the levels of soluble adhesion molecules were observed in patients with different clinical forms of schistosomiasis (Secor et al. 1994). Patients with high egg-secretion or hepatosplenic disease had significantly higher levels of s(oluble)ICAM-1 than individuals with low egg-secretion or intestinal disease. However, patients with intestinal disease had significantly higher levels of sE-selectin in their sera than did patients with hepatosplenic disease. No correlation could be found between the level of sICAM-1 and the schistosomiasis serological titre (Lejoly-Boisseau et al. 1996). Likely, higher levels of sICAM-1 reflect more intense inflammation in certain clinical forms, similarly as the correlation seen with severity in patients with primary biliary cirrhosis (Thomson et al. 1993). Malaria, often present in schistosomiasis endemic regions is a well know cause of elevated levels of sICAM1 (Jakobsen et al. 1994). However patients infected with schistosomiasis, living in a malaria non-endemic zone also have higher levels of sICAM-1 when compared with healthy individuals (LejolyBoisseau et al. 1996).

\section{ADHESION MOLECULES IN SCHISTOSOMAL GRA- NULOMOGENESIS}

Granulomatous inflammation is one of the most complex cellular immune responses requiring recruitment and activation of a variety of bloodstream cells including monocytes, lymphocytes, neutrophils, and eosinophilic granulocytes. Cell adhesion between endothelial cells and leukocytes and between leukocytes mutually is a key event in the orchestration of cellular interactions which lead to the development of a circumscribed inflammatory infiltrate know as a granuloma.

The adhesion molecules ICAM-1 and VCAM1 are both expressed in $S$. mansoni egg granulomas (Rathore et al. 1996, Jacobs et al. 1997b,c), an observation consistent with the data of Lukacs et al. (1994), who could demonstrate an increase of ICAM-1 mRNA when eggs were injected in the lungs of mice to induce granulomas. Passive immunization of mice with anti-ICAM-1 monoclonal antibodies resulted in attenuation of granuloma development and reduced the proliferative response of spleen lymphocytes towards soluble egg antigens. However, whereas ICAM-1 is constitutively expressed in normal liver tissue, VCAM-1 is not (Rathore et al. 1996); VCAM-1 is dramatically induced with the onset of the hepatic egg deposition. Strong up-regulation of ICAM-1, its ligand molecule LFA-1, and the b integrin VLA-4, but 
not VLA-6 are seen in schistosome granulomas (Langley \& Boros 1995, Jacobs et al. 1997b,c). Interaction between ICAM-1 and LFA-1 appears to be the predominant interaction in schistosome egg granuloma formation, but when the ICAM-1 gene is knocked-out, up-regulation of VCAM-1 is seen and granuloma formation is preserved (Ritter \& McKerrow 1996). VCAM-1 mediates adhesion of mononuclear to endothelial cells via binding to VLA-4 (Henseleit et al. 1994). Blockade of ICAM1, LFA-1, and VLA-4 by monoclonal antibodies markedly inhibits spleen and granuloma cell interleukin (IL) 2 and IL4 production - both cytokines being crucial for the initial events of schistosomal granuloma formation (Lukacs \& Boros 1993, Wynn et al. 1993) - as well as lymphoproliferative responses, both in the acute and chronic infection (Langley \& Boros 1995). As infection ages, granulomas appear with a non-homogenous ICAM-1/LFA-1 staining pattern, in contrast to homogenous staining granulomas during acute infection (Jacobs et al. 1997b). Whether this is due to the appearance of new cellular granuloma constituents that lack ICAM-1 or LFA-1 expression or that cellular expression is switched off in aging granulomas of previously immunoreactive cells is unclear, but since Langley and Boros (1995) did not observe apparent differences in the levels of expression of ICAM-1 and LFA-1 between acute- and chronic-infection granuloma derived cells, the appearance of new non-ICAM-1/LFA-1 immunoreactive cells in the granuloma co-existing with resident immunoreactive granuloma cells is most likely.

It is remarkable that hepatocytes become ICAM-1 positive in some forms of hepatic inflammatory damage such as alcoholic hepatitis (Burra et al. 1992), acute viral hepatitis B (Volpes et al. 1990), and liver allograft rejection (Adams et al. 1989), but not in acute or chronic hepatic schistosomiasis (Jacobs et al. 1997a,b). We could not detect hepatocyte ICAM-1 reactivity by immunohistochemistry nor by confocal microscopy. Using immunogold electron microscopy we were only able to demonstrate the presence of ICAM-1 on the microvilli of the hepatocyte cell membrane facing Disse's space (Jacobs et al. 1997b). Noteworthy is that all of these pathologies - except schistosomiasis - are the result of an immune aggression against the hepatocyte. ICAM-1-positive hepatocytes may thus be involved in facilitating the movements and possibly the "guiding" of cytotoxic lymphocytes towards hepatocytes (Volpes et al. 1990). Hepatic failure in schistosomiasis results from progressing liver fibrosis rather than immunemediated hepatocyte damage. Furthermore, it should also be noted that histologically hepatocytes are in close relationship with ICAM-1 immunoreactive sinusoidal lining cells. Activated (sinusoidal) endothelial cells can shed ICAM-1 molecules as was demonstrated by Leeuwenberg et al. (1992). Secor et al. (1994) demonstrated elevated levels of soluble ICAM-1 molecules in the serum of schistosomiasis patients. ICAM-1 molecules thus might be released along the luminal side of the sinusoidal lining cells into the bloodstream where they can be detected as soluble ICAM-1 molecules. When they are released into Disse's space, mechanical trapping in the hepatocyte microvilli may occur (Jacobs et al. 1997b).

We also observed upregulation of ICAM-1, LFA-1, and VLA-4 expression in ileal and colonic granulomas of $S$. mansoni infected mice (Jacobs et al. 1998). Morphologically, these observations could not explain the differences in granuloma size and down-modulation during chronic infection observed in these organs. The same set of adhesion molecules were also involved in the formation of hepatic (murine) S. haematobium granulomas (Jacobs et al., unpublished results). In addition, we could observe that $S$. mansoni $i$ worm-derived antigens induced ICAM-1/LFA-1-mediated granuloma formation in vivo (Jacobs et al. 1997a). Increased and early tissue expression of ICAM-1 was also seen in the skin of mice multiply infected with $S$. mansoni or vaccinated with g-irradiated cercariae after cercarial challenge (Ramaswani et al. 1997). Antigens derived from different parasite-stages or different schistosome parasites thus are capable of inducing increased localized expression of adhesion molecules in various organs of the host.

S. mansoni granuloma T-cells express the same set of adhesion molecules (L-selectin ${ }^{\text {low }}, \mathrm{CD} 44^{\text {high }}$, ICAM-1, LFA-1, and VLA-4) as granuloma T-lymphocytes in Mycobacterium avium infection (Sacco et al. 1996). Thus both Th1 (M. avium) and Th2 (S. mansoni) immune responses can give rise to granuloma infiltrating T-cells with the same adhesion molecule phenotype. This suggests that the mechanism for targeting immune cells to inflammatory sites is common to a wide variety of antigens. Schistosomal granuloma T-cells do not express the same set of adhesion molecules as splenic T-cells from S. mansoni infected animals (Sandor et al. 1995, Sacco et al. 1996). Their phenotype suggests that granuloma T-cells are almost exclusively memory/effector T-cells.

Increased expression of ICAM-1 is preceded by the production of the pro-inflammatory cytokine TNF (tumour necrosis factor)-a. TNF-a has been identified as an important mediator of granuloma formation since it restores schistosomal granuloma formation in otherwise anergic SCID mice (Amiri 
et al. 1994). Lukacs et al. (1994) demonstrated that sTNFR:Fc constructs, which are effective in inhibiting TNF-mediated events, diminished schistosome granuloma formation and lymphocyte activation in vivo. Reduced schistosome granuloma formation was also seen in mice injected with antiTNF antibodies (Joseph \& Boros 1993). One of the mechanisms of TNF-a involvement in delayed type hypersensitivity schistosome granuloma formation is by up-regulation of TNF-a inducible ICAM-1 expression (Lukacs et al. 1994), which in turn perpetuates the antigen-specific response.

APCs (antigen presenting cells), in addition to presenting processed antigen, also provide accessory cell-bound co-stimulatory signals that are necessary for stimulating maximal lymphokine production by CD4+ $\mathrm{T}$ cells. Co-stimulation is provided by a number of cell surface bound molecules such as MHC molecules, ICAM-1, and B7 molecules. Recent observations demonstrated that cells expressing the co-stimulatory molecule MHC class II are present in acute and chronic granulomas (Rathore et al. 1996) and that egg antigen-induced granuloma formation will fail to develop in MHC class II knock-out mice, but not in MHC I deficient mice because MHC class II deficient mice were unable to react to schistosome egg (Hernandez et al. 1997). These observations demonstrate that schistosomal granulomogenesis is mediated by MHC II binding CD4+ Thelper cells and that MHC I binding CD8+ T cells play little role. Antibodies directed against B7-2 but not against B7-1 accessory molecules strongly inhibited egg-induced pulmonary granuloma formation and CD4 gene expression for IL4, 5, and 13 (Subramanian et al. 1997). Acute granulomas are rich in accessory (B72 antigen positive) cells that support helper T-cell activation, but as the infection becomes more chronic, more B7-2-poor cells appear in the granulomas and these cells are capable of inducing $\mathrm{T}$ helper cell unresponsiveness (Rathore et al. 1996). Thus cellular interactions in acute granulomas may amplify egg-induced pathology, whereas in chronic granulomas they may precipitate down-modulation (Fig.). Increased expression of co-stimulatory B72 molecules is also observed in CBA/J mice displaying the more severe hepatosplenomegaly syndrome during chronic S. mansoni infection as compared to "moderate splenomegaly syndrome mice" (Freeman et al. 1996). Mice deficient for the B7/2 counter receptor generated an impaired Th2 cell response to S. mansoni infection (King et al. 1996). Downregulation of MHC class II and B7/2 molecules by IL10 results in downmodulation of egg antigen-specific delayed hypersensitivity and egg granuloma formation (Flores Villanueva et al. 1994, 1996) which induces a state of anergy that trans-

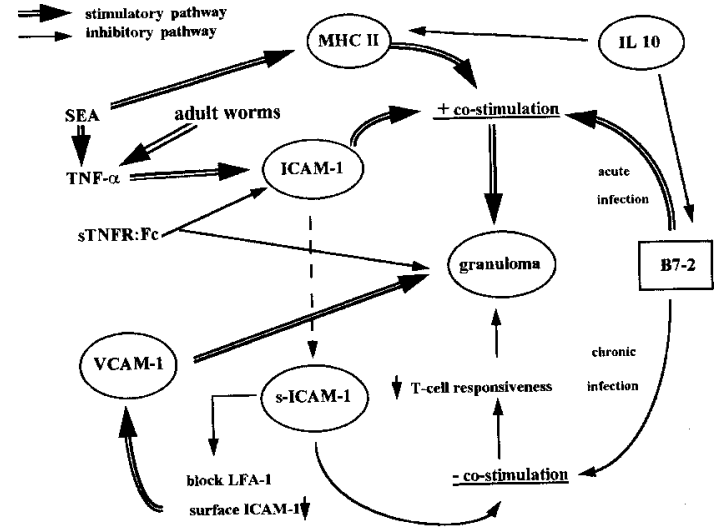

Involvement of adhesion and co-stimulatory molecules in the genesis of the Schistosoma mansoni granuloma.

lates into the formation of downmodulated granulomas as typically observed during evolving schistosomal disease (Flores Villanueva et al. 1993).

The presence of lacto-N-fucopentaose III (LNFP-III), an oligosaccharide which contains the immunogenic LewisX trisaccharide was demonstrated on soluble egg antigens (Ko et al. 1990, Koster \& Strand 1994). LewisX acts a ligand molecule for selectins (Larsen et al. 1990). LNFP-III induces proliferation of splenic B-lymphocytes and induces spleen B-cells of $S$. mansoni infected mice to produce IL-10 and PGE2 and thus down-modulate Th1 cells during experimental schistosomiasis (Velupillai \& Harn 1994). Interaction between LNFP-III and B-lymphocytes could be mediated by a fucose-dependant ligand. One such receptor is P-selectin. Although P-selectin has not been found on B-cells (Springer \& Lasky 1991), binding with a yet undescribed selectin is not excluded (Velupillai \& Harn 1994). Alternatively LNFP-III may induce proliferation of activated B-cells through cross-linking with surface ICAM-1 on Bcells. This latter hypothesis is supported by the observation that LNFP-III is present on the $a$ and b chain of LFA-1 (Forsythe et al. 1989), which is a ligand molecule for ICAM-1.

Trottein et al. (1997) demonstrated that selectin carbohydrates were involved in antibody-dependent cell-mediated cytotoxicity of macrophages to schistosomula targets. Selectin-like counter receptors could be found on the surface of developing schistosomes. It is tempting to draw parallels between leukocyte-endothelium binding through adhesion molecules and the interaction between schistosome eggs with the vascular endothelium. Although there may be specific responses of the host endothelium to the $S$. mansoni egg, the endothelial egg interaction mediated by adhesion molecules appears to be generic until proven otherwise (File 1995). 


\section{ADHESION MOLECULES AND GRANULOMA MODU- LATION}

Released soluble forms of adhesion molecules may have biological functions. Recombinant adhesion molecules have been demonstrated to block adhesion in vitro and in vivo (Watson et al. 1991). Locally high concentrations of shed adhesion molecules may thus impede adhesive interactions and provide a mechanism for negative feedback. Lselectin, but not of P- and E-selectin was observed immunohistochemically on the surface membranes of miracidia in tissue-trapped S. mansoni and $S$. japonicum eggs. El Ridi et al. (1996) suggested that circulating host-soluble L-selectin molecules, shed by L-selectin-positive leukocytes, transverse through the ultramicroscopic pores in the egg shell. These soluble L-selection molecules bind with a LewisX trisaccharide target ligand (Berg et al. 1992, Green et al. 1992). LewisX molecules are part of schistosome egg antigens (Ko et al. 1990, Koster \& Strand 1994). The binding of sL-selectin to LewisX molecules in the schistosome egg may result in complex formation that ultimately impedes the release of soluble egg antigens and hence result in down-modulation of hepatic granuloma formation observed during chronic infection (El Ridi et al. 1996). An alternative explanation for the presence of L-selectin molecules on the miracidia may be that the parasite expresses host-like molecules, allowing it to escape the immune attack of the host.

A striking feature is the inverse correlation between sICAM-1 levels in schistosomiasis patients and $\mathrm{PBMC}$ proliferative responses to egg antigens (Secor et al. 1994). Secor et al. (1994) formulated an interesting hypothesis linking sICAM-1 molecules with immune modulation of the hepatic schistosomal granuloma. Efficient activation of Tcells by APCs requires not only antigen presentation by APCs MHC class II molecules to the helperT cell TCR, but also accessory signals mediated by co-stimulatory molecules and their ligands on APCs and T-helper cells. ICAM-1 plays an important role as a co-stimulatory molecule (van Seventer et al. 1990, Kuhlman et al. 1991). Therefore, if antigen presenting cells shed ICAM-1, they may not express sufficient levels of cell surface ICAM-1 for efficient co-stimulation of SEA-specific T-cells. Further, the shed ICAM-1 molecules can block proper co-stimulation by competitively binding to LFA-1 and thereby blocking the interaction of cellular ICAM-1 with its ligand. Either or both of these mechanisms would lead to reduced co-stimulation in the granuloma or draining lymph nodes and lead to inappropriate egg antigen presentation. Consequently, sICAM-1 released through the granuloma inflammatory response could provide a mechanism for feedback control of SEA-specific T-cell responsiveness, resulting in granuloma modulation (Fig.).

Based on the currently available data it can be stated that in acute granuloma formation ICAM-1, LFA-1, VLA-4, and VCAM-1 are up-regulated, hence promoting cell-cell and cell-matrix interactions necessary for leukocyte activation and recruitment. The resulting juxtaposition enables interaction to occur between schistosome egg antigen laden macrophages (Bogers et al. 1996) bearing MHC class II molecules and SEA-specific CD4+ T-helper cells with the induction of IL2 and IL4 secretion. The presence of soluble ICAM-1 molecules, resulting in reduced levels of surface ICAM-1 and blocking of the LFA-1 ligand and the relative short-lived expression of B7-2 accessory molecules during acute infection may precipitate down-modulation of granuloma formation during chronic infection.

\section{REFERENCES}

Adams DH, Hubscher SG, Shaw J, Rothlein R, Neuberger JM 1989. Intercellular adhesion molecule-1 on liver allograft during rejection. Lancet ii: 1122-1124.

Amiri P, Locksley RM, Parslow TG, Sadick M, Rector E, Ritter D, McKerrow JH 1994. Tumor necrosis factor-a restores granulomas and induces parasite egg laying in schistosome-infected SCID mice. $\mathrm{Na}$ ture 356: 565-566.

Berg EL, Magnani J, Warnock RA, Robinson MK, Butcher EC 1994. Comparison of L-selectin and Eselectin ligand specificities: the L-selectin can bind the E-selectin ligands sialyl $\mathrm{Le}^{\mathrm{x}}$ and sialyl $\mathrm{Le}^{\mathrm{a}}$. Biochem Biophys Res Commun 184: 1048-1055.

Bogers JJ, Nibbeling HA, Deelder AM, Van Marck EA 1996. Immunohistochemical and ultrastructural localization of Schistosoma mansoni soluble egg antigens processed by the infected host. Parasitology 112: 537-543.

Bührer C, Berlin C, Thiele HG, Hamann A 1990. Lymphocyte activation and expression of the human leukocyte-endothelial cell adhesion molecule-1 (Leu8/ TQ1 antigen). Immunology 71: 442-448.

Burra P, Hubscher SG, Shaw J, Elias E, Adams DH 1992. Is the intercellular adhesion molecule-1/leukocyte function associated antigen-1 pathway of leukocyte adhesion involved in the tissue damage of alcoholic hepatitis? Gut 33: 268-271.

Camerini D, James SP, Stamenkovic I, Seed B 1989. Leu8/TQ1 is the human equivalent of the Mel-14 lymph node homing receptor. Nature 342: 78-82.

Colley DG 1975. Immune response to a soluble schistosomal egg antigen preparation during the chronic primary infection with Schistosoma mansoni. J Immunol 115: 150-156.

El Ridi R, Velupillai P, Harn DA 1994. Regulation of schistosome egg granuloma formation: host-soluble L-selectin enters tissue-trapped eggs and binds to 
carbohydrate antigens on surface membranes of miracidia. Infect Immun 64: 4700-4705.

File S 1995. Interaction of schistosome eggs with vascular endothelium. J Parasitol 81: 234-238.

Flores Villanueva PO, Chikunguwo SM, Harris TS, Stadecker MJ 1993. Role of IL-10 on antigen-presenting cell function for schistosomal egg-specific monoclonal $\mathrm{T}$ helper cell responses in vitro and in vivo. J Immunol 151: 3192-3198.

Flores-Villanueva PO, Reiser H, Stadecker MJ 1994. Regulation of Thelper cell responses in experimentel murine schistosomiasis by IL-10. Effect on expression of B7 and B7-2 costimulatory molecules by macrophages. J Immunol 153: 5190-5199.

Flores-Villanueva PO, Zheng XX, Strom TB, Stadecker MJ 1996. Recombinant IL-10 and IL-10/Fc treatment down-regulate egg antigen-specific delayed hypersensitivity reactions and granuloma formation in schistosomiasis. J Immunol 156: 3715-3720.

Forsyth KD, Simpson AC, Levinsky RJ 1989. CD15 antibodies increase neutrophil adhesion to endothelium by an LFA-1-dependent mechanism. Eur J Immunol 19: 1331-1334.

Freeman GJ, Borriello F, Hodes RJ, Reiser A, Gribben JG, Ng JW, Kim J, Goldberg JM, Hathcock K, Laszlo G, Lombard LA, Wang S, Gray GS, Nadler LM, Sharpe AM 1993. Murine B7-2, an alternative CTLA4 counter-receptor that costimulates T cell proliferation and interleukin 2 production. $J$ Exp Med 178: 2185-2192.

Freeman GL Jr, Montesano MA, Secor WE, Colley DG, Howard MJ, Bosshardt SC 1996. Immunopathogenesis and immunoregulation in schistosomiasis. Distinct chronic pathologic syndromes in $\mathrm{CBA} / \mathrm{J}$ mice. Ann NY Acad Sci 797: 151-165.

Green PJ, Tamatani T, Watanabe T, Miyasaka M, Hasegawa A, Kiso M, Yuen CT, Stoll MS, Feizil T 1992. High affinity binding of the leukocyte adhesion molecule L-selectin to 3'-sulphated-Le ${ }^{\mathrm{a}}$ and $\mathrm{Le}^{\mathrm{x}}$ oligosaccharides and the predominance of sulphate in this interaction demonstrated by binding studies with a series of lipid-linked oligosaccharides. Biochem Biophys Res Commun 188: 244-251.

Grzych JM, Pearce E, Cheever A, Caulada ZA, Caspar P, Heiny S, Lewis F, Sher A 1991. Egg deposition is the major stimulus for the production of Th2 cytokines in murine schistosomiasis mansoni. $J$ Immunol 146: 1322-1327.

Henseleit U, Steinbrink K, Sunderkotter C, Goebeler M, Roth J, Sorg C 1994. Expression of murine VCAM1 in vitro and in different models of inflammation in vivo. Exp Dermatol 3: 249-256.

Hernandez HJ, Wang Y, Tzellas N, Stadecker MJ 1997. Expression of class II, but not class I, major histocompatibility complex molecules is required for granuloma formation in infection with Schistosoma mansoni. Eur J Immunol 27: 1170-1176.

Jacobs W, Bogers J, Adriaensen D, Timmermans JP, Deelder A, Van Marck E 1997a. In vivo evaluation of adhesion molecule induction and fibrotic potential of Schistosoma mansoni adult worm antigens using confocal laser scanning microscopy. Cell Vi- sion - J Analyt Morphol 4: 97-98.

Jacobs W, Bogers J, Deelder A, Van Marck E 1997b. Expression of intercellular adhesion molecule-1 and lymphocyte function-associated antigen-1 in experimental Schistosoma mansoni infection and in synchronous periparticular hepatic granulomas in mice: immunohistochemistry, confocal laser scanning microscopy, and immunoelectron microscopy. Parasitol Res 83: 405-412.

Jacobs W, Bogers J, Deelder A, Wéry M, Van Marck E 1997c. Adult Schistosoma mansoni worms positively modulate soluble egg antigen-induced hepatic granuloma formation in vivo. Am J Pathol 150: 2033-2045.

Jacobs W, Bogers J, Timmermans JP, Deelder A, Van Marck E 1998. Adhesion molecules in intestinal Schistosoma mansoni infection. Parasitol Res 84: 276-280.

Jacobsen PH, Morris-Lones S, Ronn A, Hviid L, Theander TG, Elhassan IM, Bygbjerg IC, Greenwood B 1994. Increased plasma concentrations of sICAM-1, sVCAM-1 and SELAM-1 in patients with Plasmodium falciparum and $P$. vivax malaria and association with disease severity. Immunology 83: 665-669.

Johnston GI, Cook RG, McEver RP 1989. Cloning of GMP-140, a granule membrane protein of platelets and endothelium: sequence similarity to proteins involved in cell adhesion and inflammation. Cell 56: 1033-1044.

Joseph AL, Boros DL 1993. Tumour necrosis factor plays a role in Schistosoma mansoni egg-induced granulomatous inflammation. J Immunol 151: 5461-5471.

King CL, Xianli J, June CH, Abe R, Lee KP 1996. CD28 deficient mice generate an impaired $\mathrm{Th} 2$ response to Schistosoma mansoni infection. Eur J Immunol 26: $2448-2455$.

Ko AI, Drager UC, Harn DA 1990. A Schistosoma mansoni epitope recognized by a protective monoclonal antibody is identical to the stage-specific embryonic antigen 1. Proc Natl Acad Sci USA 87: 4159-4163.

Koster B, Strand M 1994. Schistosoma mansoni: immunolocalization of two different fucose-containing carbohydrate epitopes. Parasitology 108: $433-$ 446.

Kuhlman P, Moy VT, Lollo BA, Brian AA 1991. The accessory function of murine intercellular adhesion molecule-1 in T lymphocyte activation. Contributions of adhesion and co-activation. J Immunol 146: 1773-1782.

Langley JG, Boros DL 1995. T-lymphocyte responsiveness in murine schistosomiasis mansoni is dependant upon adhesion molecules intercellular adhesion molecule-1, lymphocyte function-associated antigen1 and very late antigen-4. Infect Immun 63: 39803986.

Larsen E, Palabrica T, Sajer S, Gilbert GR, Wagner DD, Furie BC, Furie B 1990. PADGEM-dependent adhesion of platelets to monocytes and neutrophils is mediated by a lineage-specific carbohydrate, LNF III (CD15). Cell 63: 467-474.

Leeuwenberg JF, Smeets EF, Neefjes JJ, Shaffer MA, 
Cinek T, Jeunhomme TM, Ahern TJ, Buurman WA 1992. E-selectin and intercellular adhesion molecule1 are released by activated human endothelial cells in vitro. Immunology 77: 543-549.

Lejoly-Boisseau H, Blann AD, Seigneur M, TribouleyDuret J, Tribouley J, Boisseau MR 1996. Endothelium activation related increase of soluble adhesion molecule-1 in human schistosomiasis. Lack of correlation with serology. Eur J Clin Invest 26: 522523.

Lukacs NW, Boros DL 1993. Lymphokine regulation of granuloma formation in murine schistosomiasis mansoni. Clin Immunol Immunopathol 68: 57-63.

Lukacs NW, Chensue SW, Strieter RM, Warmington K, Kunkel SL 1994. Inflammatory granuloma formation is mediated by TNF-a-inducible intercellular adhesion molecule-1. J Immunol 152: 5883-5889.

Osborn L, Hession C, Tizard R, Vassallo C, Luhowskyj S, Chi-Rosso G, Lobb R 1989. Direct expression cloning of vascular cell adhesion molecule 1 , a cytokine-induced endothelial protein that binds to lymphocytes. Cell 59: 1203-1211.

Pearce EJ, Caspar P, Grzych JM, Lewis FA, Sher A 1991. Downregulation of Th1 cytokine production accompanies induction of Th2 responses by a parasitic helminth, Schistosoma mansoni. J Exp Med 173: 159-166.

Pigott R, Dillon LP, Hemingway IH, Gearing AJ 1992. Soluble forms of E-selectin, ICAM-1 and VCAM-1 are present in the supernatants of cytokine activated cultured endothelial cells. Biochem Biophys Res Commun 187: 584-589.

Ramaswamy K, Yi-Xun He, Salafsky B 1997. ICAM-1 and iNOS expression increased in the skin of mice after vaccination with g-irradiated cercariae of Schistosoma mansoni. Exp Parasitol 86: 118-132.

Rathore A, Sacristan C, Ricklan DE, Flores Villanueva PO, Stadecker MJ 1996. In situ analysis of B7-2 costimulatory, major histocompatibility complex class II, and adhesion molecule expression in schistosomal egg granulomas. Am J Pathol 149: 187194.

Ritter DM, McKerrow JH 1996. Intercellular adhesion molecule 1 is the major adhesion molecule expressed during schistosome granuloma formation. Infect Immun 64: 4706-4713.

Rothlein R, Mainolfi EA, Czajkowski M, Marlin SD 1991. A form of circulating ICAM-1 in human serum. J Immunol 147: 3788-3793.

Sacco RE, Jensen RJ, Thoen CO, Sandor M, Weinstock J, Lynch RG, Dailey MO 1996. Cytokine secretion and adhesion molecule expression by granuloma $\mathrm{T}$ lymphocytes in Mycobacterium avium infection. Am J Pathol 148: 1935-1948.

Sandor M, Sperling AI, Cook GA, Weinstock JV, Lynch
RG, Bluestone JA 1995. Two waves of gd T cells expressing different $\mathrm{Vdgenes}$ are recruited into schistosome-induced liver granulomas. J Immunol 155: 275-284.

Secor WE, dos Reis MG, Ramos EA, Matos EP, Reis EA, do Carmo TM, Harn Jr DA 1994. Soluble intercellular adhesion molecules in human schistosomiasis: correlations with disease severity and decreased responsiveness to egg antigens. Infect Immun 62: 2695-2701.

Springer TA 1990. Adhesion receptors of the immune system. Nature 346: 425-434.

Springer TA, Lasky LA 1991. Cell adhesion. Sticky sugars for selectins. Nature 349: 196-197.

Subramanian G, Kazura JW, Pearlman E, Jia X, Malhotra I, King CL 1997. B7-2 requirement for helminthinduced granuloma formation and CD4 type $2 \mathrm{~T}$ helper cell cytokine expression. J Immunol 158: 5914-5920.

Thomson AW, Satch S, Tamura K, Woo J, Gavaler J, van Theil DH 1993. Circulating ICAM-1 in primairy biliary cirrhosis: correlations with disease severity. J Immunol 150: 306A.

Trottein F, Nutten S, Papin JP, Leportier C, PoulainGodefroy O, Capron A, Capron M 1997. Role of adhesion molecules of the selectin-carbohydrate families in antibody-dependent cell-mediated cytotoxicity to schistosome targets. J Immunol 159: 804-811.

van Seventer GA, Shimizu Y, Horgan KJ, Shaw S 1990. The LFA-1 ligand ICAM-1 provides an important co-stimulatory signal for $\mathrm{T}$ cell receptor-mediated activation of resting T cells. J Immunol 144: 45794586.

Velupillai P, Harn DA 1994. Oligosaccharide-specific induction of interleukin 10 production by $\mathrm{B} 220^{+}$cells from schistosome-infected mice: a mechanism for regulation of $\mathrm{CD}^{+}{ }^{+}$T-cell subsets. Proc Natl Acad Sci USA 91: 18-22.

Volpes R, Van Den Oort JJ, Desmet VJ 1990. Hepatic expression of intercellular adhesion molecule-1 (ICAM-1) in viral hepatitis B. Hepatology 12: 148154.

Watson SR, Fennie C, Lasky LA 1991. Neutrophil influx into an inflammatory site is inhibited by a soluble homing receptor chimera. Nature 349: 164-167.

WHO Expert Committee 1993. The control of schistosomiasis. WHO Techn Rep Ser 830: 1-86.

Wynn TA, Cheever AW 1995. Cytokine regulation of granuloma formation in schistosomiasis. Curr Opin Immunol 7: 505-511.

Wynn TA, Eltoum I, Cheever AW, Lewis FA, Gause WC, Sher A 1993. Analysis of cytokine mRNA expression during primary granuloma formation induced by eggs of Schistosoma mansoni. J Immunol 151: 1430-1440. 
530 Adhesion and Co-stimulatory Molecules in Schistosomiasis - W Jacobs, E Van Marck 